\title{
Splotch locus mouse mutants: models for neural tube defects and Waardenburg syndrome type I in humans
}

\author{
Connie E Moase, Daphne G Trasler
}

The majority of data that contribute to our understanding of mammalian embryonic development are obtained from the study of animal models. An increasing availability of molecular tools to dissect these model systems has enabled us to establish parallels with human development that range from morphogenetic similarities to homologous DNA sequences. The mouse mutant splotch $(S p)$ has long been recognised as a model for human neural tube defects (NTDs) and, more recently, has become a candidate model for Waardenburg syndrome type I (WSI) in humans. ${ }^{12}$ This latter circumstance is based on similarities in some of the defects in neural crest cell (NCC) derivatives that are shared by $S p$ mutants and Waardenburg patients, as well as the possibility that WSI may be localised to human chromosome 2q37, a region known to share homologies with mouse chromosome 1 where the $S p$ locus is found.

Neurulation occurs between gestation day 8.5 and late day 9 in the mouse (plug day = day 0 ) and this is comparable to days 20 to 26 of human embryonic development. ${ }^{3}$ Neural crest cells originate from the neural tube and begin to emigrate just before cephalic neural tube closure, or shortly after trunk neurulation in mammalian embryos. Thus, the two events occur almost simultaneously in development. NCCs then migrate to various regions of the body and differentiate into a variety of structures including neuronal cells of the sensory and autonomic ganglia, nerve supporting structures, mesoectodermal structures, certain cells of the endocrine system, and melanocytes. ${ }^{45}$ The fact that both neurulation and NCC emigration are disrupted in the $S p$ mutant makes this a useful model for understanding the basis of two fundamental developmental processes.

This article reviews the results of investigations that have examined splotch locus mutants. These studies include histological analyses at the structural and ultrastructural levels, gene-teratogen interactions, as well as outcomes from immunohistochemical, biochemical, cellular, and molecular approaches. Information gathered from the analyses of these mutants has contributed to our understanding of neurulation and neural crest cell emigration, and has provided clues as to how these two fundamental processes may be developmentally related.

\section{Splotch locus alleles}

ORIGIN OF SPLOTCH LOCUS MUTANTS

Splotch locus mutants include several allelic variants that serve as mouse models for both NTDs and deficiencies in NCC derivatives. The original splotch mutation arose spontaneously on a C57BL inbred background and was first described by Russell. ${ }^{6}$ Since then, Dickie $^{7}$ reported other de novo splotch mutations $\left(S p^{7}, S p^{2 f}\right.$, and $\left.S p^{37}\right)$, as well as the appearance of an allele splotch delayed $\left(S p^{d}\right)$. More recently, Beechey and Searle ${ }^{8}$ described three radiation induced mutations at this locus, two of which appeared to be the same as the original $S p$ mutation $\left(S p^{I H}\right.$ and $\left.S p^{2 H}\right)$, and a third was referred to as splotch retarded $\left(S p^{r}\right)$, an allele of $S p$ and $S p^{d}$.

Early linkage analysis showed $S p$ belonged to linkage group XIII and was positioned between the coat colour mutation leaden $(l n)$ and the hair follicle mutation fuzzy $(f z) . .^{910}$ Recombination frequencies between $S p^{d}$ and either $\ln$ or $f z$ were found to be similar to those of $S p,{ }^{7}$ supporting the idea that $S p^{d}$ was indeed allelic to $S p$. Since then, the splotch locus has been mapped more specifically to band $\mathrm{C} 4$ of chromosome $1^{11}$ and investigations to define this locus further using DNA probes are currently in progress.

\section{HOMOZYGOUS FEATURES}

Mutations at the $S p$ locus are classified as semidominant lethal, ${ }^{7}$ with differences in homozygous phenotype being the factor that distinguishes between splotch and splotch delayed. Most $S p$ homozygotes develop spina bifida (lumbosacral rachischisis) and over half exhibit exencephaly (cranioschisis) as well, owing to lack of closure in the hindbrain region. ${ }^{12}$ On occasion, these mutants may develop only a tail flexion defect that results in a curly tail. ${ }^{13}$ However, all mutants die in utero at approximately 13 or 14 days of gestation. ${ }^{12}$ This is in contrast to the $S p^{d}$ mutant which develops only spina bifida and survives until birth, hence undergoing delayed death. ${ }^{7}$

Unlike $S p$ and $S p^{d}$, splotch retarded homozygotes are presumed to die before implantation. ${ }^{8}$ This mutation is more severe than $S p$ or $S p^{d}$ as it involves a cytogenetically detectable deletion of the $1 \mathrm{C} 4$ band, which constitutes approximately $2 \%$ of the total physical length of chromosome $1 .{ }^{11}$ 


\section{HETEROZYGOUS PHENOTYPE}

$S p, S p^{d}$, and $S p^{r}$ heterozygotes display a similar phenotype that is characterised by a white belly patch, feet, and tail tip, although $S p$ heterozygotes occasionally lack the ventra spotting. This pigmentation defect results from the failure of neural crest cells to populate these regions sufficiently during development. ${ }^{12}$ Other NCC derived structures have been shown to be deficient or absent in both $S p$ and $S p^{d}$ mutants as well. These include spinal ganglia, ${ }^{12}$ Schwann cells, ${ }^{15}$ and NCC derived structures of the heart. ${ }^{1617}$ What distinguishes the $S p^{r}$ heterozygote from $S p$ and $S p^{d}$ gene carriers is the fact that it experiences an overall growth retardation which persists throughout adulthood. ${ }^{8}$ However, because this particular mutant has only recently become available, it has not been characterised as extensively as $S p$ or $S p^{d}$.

PHENOTYPIC VARIATIONS

Variations in expressivity have been obtained by outcrossing $S p$ or $S p^{d}$ gene carriers to mice with different genetic backgrounds. Using $S p^{d}$ on a heterogeneous BALB/c stock, Kalter ${ }^{18}$ showed that each of three teratogens known to cause exencephaly could do so at a higher frequency in $S p^{d}$ homozygotes than in nonmutant litter mates. Thus, even though $S p^{d}$ homozygotes do not normally express exencephaly, they appear to be liable to such an occurrence. This was further supported by the observation that exencephaly occurred at a frequency of $6 \cdot 1 \%$ in day $16 S p^{d}$ homozygous embryos from $\mathrm{F} 1$ mice that had originated by outcrossing $S p^{d}$ inbreds to an In(1)1Rk stock. ${ }^{19}$

Outcrossing $S p$ inbreds to $\operatorname{In}(1) 1 \mathrm{Rk}$ mice resulted in an altered distribution frequency of NTDs in $S p$ homozygous embryos as well. The proportions of NTDs changed from $56 \%$ of mutants having exencephaly in addition to spina bifida ${ }^{12}$ to $100 \%$ of mutant embryos having exencephaly, with spina bifida occurring only about $25 \%$ of the time. ${ }^{19}$ In addition, $S p$ mutants on this background can survive longer than the usual 13 to 14 days of gestation in an inbred line, with viable embryos being present up to day 18 of gestation. ${ }^{19}$

\section{Splotch as a model for human NTDs}

In humans, NTDs are primarily the result of interactions between genetic and environmental factors. The cellular and molecular mechanisms of neurulation have yet to be fully understood. However, one approach towards this end is to examine abnormal neural tube closure in genetic models where environmental factors can be manipulated. Various NTD mouse mutants such as loop-tail, ${ }^{2021}$ crooked, ${ }^{22}$ rib-fusion, ${ }^{23}$ and extra-toes, ${ }^{24}{ }^{25}$ express additional gross anomalies in unrelated organ systems, thus complicating the analysis of NTD pathogenesis. Another model, the curly-tail mutant, ${ }^{2627}$ is a recessive mutation with incomplete penetrance that has yet to be assigned to a particular chromosome. This, in addition to the fact that only about $60 \%$ of all curly-tail embryos develop an NTD, makes it difficult to carry out investigations on abnormal neural tube closure until the development of an NTD is well under way.

In the case of $S p$, however, only the skull and vertebral malformations that are associated with an open neural tube are present in conjunction with the defects in certain NCC derivatives, which also originate from the neural tube. Therefore, it is possible that a mechanism directly involved in neurulation is the primary target of the $S p$ mutation. This, combined with the fact that $S p$ mutants can be identified before the manifestation of an NTD, ${ }^{1928}$ make it a good model for understanding abnormal neural tube closure.

\section{Pathogenesis of splotch mutant defects}

Some of the first studies involving the splotch mutant examined the pathology of the abnormally developed neural epithelium. Hsu and Van Dyke, ${ }^{29}$ as well as Auerbach, ${ }^{12}$ reported an extensive overgrowth of neural tissue in open regions of the neural tube. Hsu and Van Dyke $^{29}$ attributed this to an increase in mitotic activity between 13.5 and 14.5 days of gestation. Using tritiated thymidine incorporation, Wilson ${ }^{30}$ showed that mesencephalic cells of day 10 and $11 S p$ embryos undergo a longer cell cycle than that found in heterozygous or wild type embryos, and that proliferation is actually decreased in these mutants. However, through analysis of even younger embryos, Kapron-Brás and Trasler ${ }^{31}$ found that the mitotic index was similar between all regions of day $9 S p$ and control embryos. Thus, any difference in the mitotic index between mutants and non-mutants probably reflects secondary changes following the initial disruption of neural tube closure.

Other histological features of abnormally developed $S p$ or $S p^{d}$ mutants include neural tube irregularities throughout the embryo, abnormal tail morphology, distortions of the brain lumen ${ }^{12}$ with significantly reduced lumen size, ${ }^{32}$ abnormal otic vesicle differentiation in those with exencephaly, ${ }^{33}$ disorganised neuroepithelial tissue with more intercellular space, ${ }^{34}$ significant reductions in the area of the neuroepithelium as well as the forebrain, ${ }^{32}$ and reduced or absent neural crest cell derivatives. ${ }^{12} 14$ However, because these observations were from embryos that already exhibited the defect, it was impossible to determine whether they were primary or secondary effects of the mutation. In order to understand the aetiology of $S p$ locus mutations, it is necessary to examine embryos before the appearance of the defect.

\section{Aetiology of splotch NTDs}

Neuropore measurements of embryos derived from intercrosses of $S p$ or $S p^{d}$ heterozygotes show that presumptive homozygous mutants have longer posterior neuropores than their non-mutant heterozygous and wild type litter 
mates. In addition, presumptive $S p$ mutants have longer anterior neuropores. ${ }^{35} 36$ This delay in neuropore closure was postulated to be the underlying factor that predisposed $S p$ gene carriers (that is, heterozygotes), which are morphologically normal, to develop NTDs upon exposure to a teratogenic agent ${ }^{3637}$ (see section on Splotch and retinoic acid, below). These results were later corroborated by using embryos that were positively identified (see section on Chromosomal markers, below) as $S p$ gene carriers. ${ }^{38}$ Similarly, this delay in posterior neuropore closure has been shown to occur in the curly-tail mutant discussed previously. ${ }^{39}$ However, the mechanism responsible for this delay in closure in any of these mutants has yet to be elucidated.

\section{Markers for splotch locus mutants GRAFTED EMBRYONIC ECTODERM}

As mentioned above, differentiation between mutant and non-mutant embryos before the expression of a malformation is necessary in order to identify potential primary causal factors of abnormal development. One method of distinguishing $S p$ mutants from their heterozygous and wild type litter mates involves isolating dorsal ectodermal tissue together with its underlying mesoderm from day 9 embryos, and implanting it into either embryonic chick coelom, or the anterior eye chamber of adult albino mice. ${ }^{12}$ Grafted ectoderm from $S p$ homozygotes fails to generate pigment after 16 days, whereas tissues from heterozygous and wild type embryos produce pigmentation. Using this technique to identify day $9 S p$ mutant embryos, Wilson and Finta ${ }^{40}$ showed the frequent occurrence of gap junctional vesicles in the lumbosacral region of the neural groove that subsequently fails to close. Gap junctional vesicles, which are normally abundant in C57B1/6J embryos a day earlier in gestation, ${ }^{41}$ are suggested to represent a breakdown of gap junctions, and thus may be an indicator of decreased cell-cell communication in the $S p$ mutant at this stage of development.

\section{CHROMOSOMAL MARKERS}

Robertsonian translocation $\mathrm{Rb}(1.3) 1 \mathrm{Bnr}$

Although $S p$ mutants can be identified by either skin grafts or the length of their neuropores relative to non-mutant embryos at the same developmental stage, it is not possible to distinguish between heterozygous and wild type embryos by these methods. Thus, it was necessary to develop a more specific marker. Kapron-Brás et $a l^{42}$ devised a breeding scheme that introduced a 1.3 Robertsonian translocation (a fusion of chromosomes 1 and 3 ) into the $S p$ line. This could then be used as a marker for the chromosome carrying the wild type allele. However, using this system to predict the genotype provided $80 \%$ reliability in heterozygotes, but only $60 \%$ accuracy in homozygotes owing to cross over. Therefore, a more dependable genotyping strategy was required.
Inversion In (1) $1 R k$

A more reliable marker was established using the In(1)1Rk mouse line which is homozygous for a paracentric inversion that spans the $S p$ locus. Using an appropriate breeding design (fig 1), Moase and Trasler $^{19}$ showed that the accuracy of this marker was greater than $98 \%$ owing to recombination suppression by the inversion. Because this inverted segment encompasses certain biochemical loci that differ in isotype from the splotch line, ${ }^{43}$ and also includes $42 \%$ of the total length of chromosome 1,44 the genotype of each individual embryo can be established by one of two methods. Individual embryos at day 11 of gestation or older can be genotyped on the basis of their isocitrate dehydrogenase profile ${ }^{19}$ (fig 2), while younger embryos can be identified using cytogenetic analysis $^{28}$ (fig 3). Until the $S p$ locus or closely linked markers are identified, this marking scheme involving the In(1) $1 \mathrm{Rk}$ inversion is the most reliable method to date for differentiating between mutant, heterozygous, and wild type embryos.

One aspect that is revealed with the use of this marker is the fact that $4 \%$ of day $16 S p^{d}$ mice, and $9 \%$ of day $9 S p$ mice which exhibit an NTD are actually heterozygotes ${ }^{19}$ (unpublished observations). Using this system, however, it is not possible to determine whether NTDs are found in inbred heterozygotes as well, or only when these mice are outcrossed to the In(1) 1 Rk stock.

\section{Gene-teratogen interactions}

Numerous studies have examined the effect of teratogens on mice that are carriers of a mutant gene. Embryos heterozygous for a recessive or semidominant mutant gene often express the homozygous phenotype when exposed to a specific teratogen. This positive effect may be indicative of possible shared mechanisms

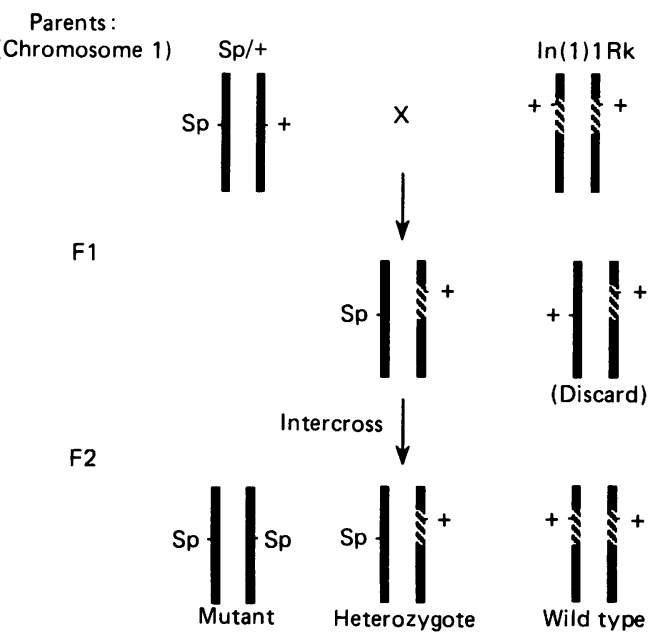

Figure 1 Breeding scheme to obtain biochemically and cytogenetically marked splotch mice (chromosome 1). The hatched region contains the cytogenetically detectable In (1) 1RK inversion, and also carries the isocitrate dehydrogenase $b\left(I d h-1^{b}\right)$ isozyme. These are markers for the wild type allele in $F 2$ mice, while solid chromosomes carry the Idh-1a isozyme, and indicate the presence of the Sp allele in F2 mice. (Adapted from presence of the Sp alle 


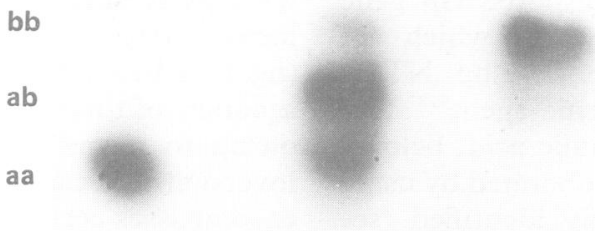

0

Figure 2 Isocitrate dehydrogenase profiles from individual day $11 S p / S p, S p /+$, and $+1+$ embryos. The slow migrating band (aa) in lane 1 is from a $S p$ homozygote, lane 2 is heterozygous for Idh-1 ${ }^{a}$ and $I d h-1^{b}(a b)$ isotypes and is from a Sp heterozygote, lan 3 contains the fast migrating variant $(b b)$ and is from $a$ $+/+$ embryo. ( $0=$ origin; from Moase and Trasler. $\left.{ }^{19}\right)$

through which mutant genes and teratogens exert their effects. Cole and Trasler ${ }^{45}$ showed that progeny obtained from a cross of either crooked or rib-fusion heterozygotes to nonmutant strains were more susceptible to NTDs after in utero exposure to teratogenic doses of insulin than were progeny from intercrosses between non-mutants. Other examples of positive interactions include 5-fluorouracil with the limb mutants luxoid and luxate, ${ }^{46}$ trypan blue and NTDs in brachyphalangy carriers, ${ }^{47}$ trypan blue or actinomycin D with tail malformations in brachyury gene carriers, ${ }^{4849}$ and 6aminonicotinamide with cleft lip in dancer heterozygotes. ${ }^{50}$

SPLOTCH AND RETINOIC ACID (RA)

$R A$ induction of NTDs

Retinoic acid (RA), a vitamin A analogue, has been extensively studied with respect to its interaction with splotch and splotch-delayed. A positive gene-teratogen interaction has been shown for carriers of the $S p$ gene in vivo ${ }^{3637}$ as well as in vitro using the whole embryo culture

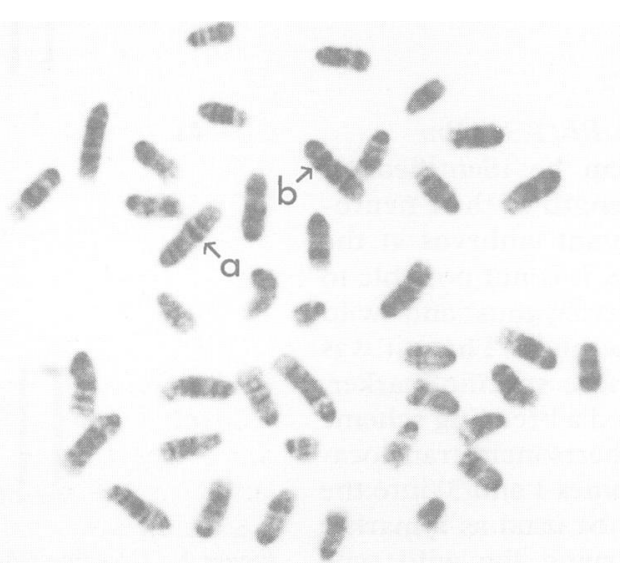

Figure 3 A chromosomal spread from a cytogenetically marked day $9 S p^{d}$ heterozygous embryo. $G$ banding shows the pattern associated with the chromosome 1 that carries the $S p^{d}$ (or $S p$ ) allele $(a)$, and the chromosome 1 derived from the In (1)1Rk line which carries the inversion and, hence, the wild type allele (b). (From Moase and Trasler. ${ }^{28}$ ) system..$^{38}$ However, no positive interaction with retinoic acid was observed for carriers of $S p^{d}{ }^{35}$ In an extensive histological analysis to determine whether $S p$ and retinoic acid were acting through the same mechanism to produce NTDs, Kapron-Brás and Trasler $^{31}$ showed that the $S p$ gene is associated with reducing both the number of NCCs released from the neural tube, and the amount of extracellular space surrounding the neural tube, including the area between the neural tube and surface ectoderm. On the other hand, retinoic acid caused disruptions in the spatial relationship between the notochord and neural tube, as well as in the shape of the neural tube. Thus, retinoic acid and the $S p$ gene affect different aspects of neurulation, but combine to induce NTDs in a greater degree than either factor by itself.

\section{$R A$ reduction of NTDs}

Although treatment of non-mutant embryos with 30 to $60 \mathrm{mg} / \mathrm{kg}$ of retinoic acid on day 8 of gestation causes NTDs, ${ }^{37}$ it was also noted that, as with the curly-tail mutant, ${ }^{51}$ low retinoic acid doses of $5 \mathrm{mg} / \mathrm{kg}$ administered one day later in gestation (day 9) significantly reduces the incidence of NTDs in litters from $S p$ heterozygote intercrosses, without increasing the resorption frequency. ${ }^{52}$ When this study was repeated using genotypically identifiable $S p^{d}$ embryos, it was found that the reduction in NTD frequency was actually attributable to retinoic acid induced selective mortality of mutant embryos, and that this induced mortality was not enough to increase the resorption frequency significantly. ${ }^{19}$ In genotypically identifiable $S p$ embryos, the effect was somewhat different. Rather than reducing the NTD frequency, these low RA doses appeared to induce NTDs in day 11 heterozygotes. However, there was a higher incidence of developmentally retarded, malformed embryos in the treated group, suggesting that even a low dose of RA was indeed detrimental to embryonic development and viability.

Studies involving the curly-tail mutant have shown that administration of the DNA inhibitor mitomycin $\mathrm{C}^{53}$ or maternal food deprivation during embryonic neurulation, ${ }^{54}$ significantly reduces the NTD frequency without increasing the resorption frequency. Although the effect of DNA inhibitors has not been tested in $S p$ locus mutants, a pilot study by Mehin and Trasler $^{69}$ indicates that food deprivation does not reduce the overall NTD frequency in litters obtained from $S p^{d} /+$ intercrosses. This, in conjunction with other differences, indicates that $S p$ and curlytail mutants do not share a common aetiological basis for NTDs other than the fact that both experience delays in neural tube closure.

\section{Splotch extracellular matrix components}

According to histological observations, ${ }^{31}$ the closed neural tube in the trunk region of $S p / S p$ 
embryos is in close apposition to the overlying ectoderm, and, unlike control sections, has very little extracellular space between the two tissues. Because fewer NCCs were observed in these regions compared to controls, it was suggested that the lack of extracellular space could be due to alterations in extracellular matrix components and that this, in turn, may inhibit NCC emigration from the neural tube.

Several immunohistochemical and ultrastructural analyses have focused on the temporal and spatial localisation of specific extracellular matrix constituents in $S p$ and $S p^{d}$ mutants. Using late day 9 or day $11 S p^{d}$ embryos that exhibited the mutant phenotype, O'Shea and $\mathrm{Liu}^{55}$ found that the basal lamina of the neuroepithelium was disorganised, and that there was less laminin and collagen IV, and more fibronectin and heparan sulphate proteoglycan (HSPG) in the dorsolateral region of the neural tube compared to controls. Fibronectin and HSPG were also found to be displaced in the mutants during secondary neurulation. McLone and Knepper ${ }^{56}$ also observed alterations in certain glycoconjugates and glycosaminoglycans in day $10 S p$ mutants. Using enzymatic digestion to quantitate particular extracellular matrix components, these authors reported that the distribution of hyaluronic acid (HA) and chondroitin sulphate (CSPG) was approximately equal in mutant neuroepithelium, whereas in normal embryos $\mathrm{HA}$ is predominant in the open neural tube and CSPG is predominant in the closed neural tube. McLone and Knepper ${ }^{56}$ also found differences in lectin staining patterns between $S p$ mutants and controls, with a persistent and intense concanavalin-A staining on the abnormal luminal surface.

Other studies have examined $S p$ and $S p^{d}$ mutant embryos at slightly earlier stages of development in order to assess extracellular matrix aspects before the abnormal phenotype is apparent. These embryos were identified as mutant by the In(1)1Rk marker. Trasler and Morriss-Kay ${ }^{57}$ observed greater amounts of both CSPG and HSPG antibody fluorescence in the neuroepithelial basement membrane of cranial and caudal sections from 5 to 15 somite stage $S p$ and $S p^{d}$ mutant embryos compared to non-mutants. In an extensive ultrastructural and morphometric analysis of caudal $S p^{d}$ mutant sections at 15 to 18 somite stage of development, Yang and Trasler $^{58}$ observed that the basal lamina of the overlying surface ectoderm was not as well formed as that of controls, that there were fewer mesodermal cells in mutant embryos, and that the neuroepithelium was disorganised and contained a greater amount of intercellular space. This latter observation corroborated similar findings in slightly older $S p$ mutants. ${ }^{34}$ Furthermore, it showed that these morphogenetic differences were present even before the defects become apparent, suggesting that one of the primary effects of the mutant gene is to elicit these changes in the neuroepithelium. In contrast, Yang and Trasler ${ }^{58}$ did not observe the significant differences in gap junctional vesicle formation between $S p^{d}$ mutants and controls that were reported by Wilson and Finta ${ }^{40}$ from their examination of early day 9 $S p$ mutants. $S p$ mutants have been shown to be more severely affected than $S p^{d}$ mutants in other respects, such as viability, type of NTD, and reductions in spinal ganglia and emigrating NCCs. Thus, it is not surprising that differences in numbers of gap junctional vesicles are observed between $S p$ and $S p^{d}$ as well.

\section{In vitro analysis of splotch NCCs}

Although NTDs occur in most embryos that are homozygous for $S p$ or $S p^{d}$, genotypic analysis has shown that $11 \%$ of mutant embryos do not exhibit such a defect. However, histological examination showed that these particular embryos did have severe reductions or absences of NCC derived spinal ganglia. ${ }^{14}$ This indicated that defects observed in NCC derivatives are not the result of NTDs in these mutants. In fact, morphometric analysis showed that even some heterozygous embryos have significantly reduced volumes of spinal ganglia compared to wild type embryos, but presumably this deficiency is not detrimental to the survival of heterozygotes. Thus, since one defect was not contingent on the others' occurrence, it was possible that neural tube closure and the release of NCCs from the neural tube may instead share a regulatory event that, if disrupted, could result in the defects seen in both developmental pathways.

Kapron-Brás and Trasler ${ }^{31}$ have shown that few NCCs are released from mutant neural tubes. However, it was unclear as to whether this occurred as a result of an abnormality in the neuroepithelium, the ECM into which NCCs emigrate, or whether the NCCs themselves were at fault. Examination of NCC emigration from explanted neural tubes ${ }^{28}$ showed that there was approximately a 24 hour delay in the release of NCCs from mutant neural tubes compared to non-mutants, with $S p$ being more severely affected than $S p^{d}$. Provision of an enriched three dimensional extracellular matrix containing laminin, collagen IV, HSPG, and entactin failed to enhance NCC release significantly, and assessment of mitotic indices showed no difference in NCC proliferation between mutant and non-mutant genotypes. Therefore, these findings indicated that the neuroepithelium from which NCCs arise may be faulty in the $S p$ mutant with respect to the mechanism involved in the release of NCCs. ${ }^{28}$

\section{Cell adhesion molecules (CAMs) in splotch}

Since specific cell adhesion molecules are known to be involved in neural development, it is of interest to examine some of these with respect to $S p$. Using immunofluorescent techniques, Moase and Trasler ${ }^{59}$ found that neuroepithelial tissue in sections from mutant embryos fluoresced with greater intensity in response to antibodies against the neural cell adhesion molecule N-CAM than did control sections. Further analysis by immunoblotting 
showed that both $S p$ and $S p^{d}$ mutants, as well as day 9 heterozygous embryos, exhibited altered N-CAM profiles compared to wild type embryos. These alterations may involve a change in the conversion of high molecular weight N-CAM to lower molecular weight forms, similar to the observation in another neurological mutant, staggerer. ${ }^{60}$ However, in the case of splotch, this difference is apparent early in development rather than postnatally. $\mathrm{N}-\mathrm{CAM}$ is coded by a gene localised to chromosome 9 in mice. Therefore, this alteration in splotch probably relates to regulation or post-translational processing of the N-CAM protein, which may or may not be directly influenced by the $S p$ locus on chromosome 1 . In view of the fact that N-CAM copurifies with certain ECM components, including HSPG and CSPG, ${ }^{6162}$ which appear necessary for N-CAM mediated cell adhesion, it is possible that an altered N-CAM species could bring about the cascade of abnormalities observed in $S p$ locus mutants. However, further investigation is necessary to substantiate this idea.

\section{Present and future directions}

In addition to investigations that analyse $S p$ locus mutations at the cellular level, progress is also being made towards defining this locus at the molecular level. Carriers of the $S p^{r}$ allele are useful in this respect owing to the large deleted segment at this locus. Four genes which fall within this deleted region have been identified ${ }^{63}$; however, these markers are present in the other allelic mutants. Thus, it is necessary to find more markers and to establish a multilocus linkage map of this region in order to ascertain homologous sequences between mouse and man. Foy et $a l^{1}$ have tentatively established linkage between Waardenburg syndrome type I and placental alkaline phosphatase, which is localised to human chromosome 2q37. These findings have been corroborated by another group that examined a single large WSI pedigree. ${ }^{2}$ This is interesting in view of the fact that chromosome $2 \mathrm{q} 37$ is known to share homologies with the region containing the splotch locus on mouse chromosome 1 . The fact that phenotypic similarities occur in splotch mice and WSI patients (that is, pigmentation disturbances and occasional NTDs in WSI subjects ${ }^{64-68}$ ) suggests that Waardenburg syndrome may be the human counterpart to the mouse mutant splotch. In addition, continuing studies involving molecular analysis of the $S p$ locus may eventually show mouse-human homologies in DNA sequences that are associated with the development of NTDs.

\section{Note added in proof}

Recently Pax-3, a murine DNA binding protein expressed during early neurogenesis was mapped to mouse chromosome $1 .{ }^{70} \mathrm{Pax}-3$ was found to be deleted in $S p^{r} /+$ mice and the $S p^{2 H}$ allele was shown to have a 32 nucleotide deletion within the paired homeo domain coding portion of Pax-3. ${ }^{71}$ Therefore, Pax-3 must have a key role in neurulation. Further, Pax-3 is the mouse homologue of the human HuP2 gene, ${ }^{72}$ which suggests the latter may map near 2q37 and may be altered in WSI.

This research was supported by Natural Sciences and Engineering Research Council (NSERC) and Medical Research Council (MRC) postgraduate studentships (CEM) as well as an MRC (Canada) research grant (DGT).

1 Foy C, Newton V, Wellesley D, Harris R, Read A. Assignment of the locus for Waardenburg syndrome type I to human chromosome $2 \mathrm{q} 37$ and possible homology to the splotch mouse. Am $\mathcal{F}$ Hum Genet 1990;46:1017-23.

2 Asher JH Jr, Morell R, Friedman TB. Waardenburg syn drome (WS): the analysis of a single family with a WSI mutation showing linkage to RFLP markers on human mutation showing linkage to RFLP markers on hun

3 Rugh R. The mouse. Its reproduction and development. Minneapolis: Burgess Publishing, 1968:304-5.

4 Weston JA. The migration and differentiation of neural crest cells. Adv Morphogenet 1970;8:41-114.

5 LeDouarin N. Migration and differentiation of neural cres cells. Curr Top Dev Biol 1980;16:31-85.

6 Russell WL. Splotch, a new mutation in the house mouse Mus musculus. Genetics 1947;32:107A.

7 Dickie MM. New splotch alleles in the mouse. 7 Hered 1964;55:97-101.

8 Beechey CV, Searle AG. Mutations at the $S p$ locus. Mouse News Letter 1986;75:28.

9 Snell GD, Dickie MM, Smith P, Kelton DE. Linkage of loop-tail, leaden, splotch and fuzzy in the mouse. Heredity 1954;8:271-3.

10 Parsons PA. A balanced four-point linkage experiment for linkage group XIII of the house mouse. Heredity 1958;12:77-95.

11 Evans EP, Burtenshaw MD, Beechey CV, Searle AG. A splotch locus deletion visible by Giemsa banding. Mouse News Letter 1988;81:66.

12 Auerbach R. Analysis of the developmental effects of a lethal mutation in the house mouse. $f$ Exp Zool 1954;127:305-29.

13 Russell WL, Gower JS. Offspring from transplanted ovaries of fetal mice homozygous for a lethal gene $(S p)$ that kills before birth. Genetics 1950;35:133.

14 Moase CE, Trasler DG. Spinal ganglia reduction in the splotch-delayed mouse neural tube defect mutant. Teratology 1989;40:67-75.

15 Franz $T$. Defective ensheathment of motoric nerves in the splotch mutant mouse. Acta Anat (Basel) 1990;138:24653.

16 Effmann EL, Whitman SA. Cardiovascular defects in murine homozygous splotch embryos. Teratology 1986;33:36C.

17 Franz T. Persistent truncus arteriosus in the splotch mutant mouse. Anat Embryol 1989;180:457-64

18 Kalter H. Experimental teratological studies with the mouse CNS mutations cranioschisis and delayed splotch. f Craniofac Genet Dev Biol 1985;1(suppl):339-42.

19 Moase CE, Trasler DG. Retinoic acid-induced selective mortality of splotch-delayed mouse neural tube defect mutants. Teratology 1987;36:335-43.

20 Strong LC, Hollander WF. Hereditary loop-tail in the house mouse accompanied by imperforate vagina and with lethal craniorachischisis when homozygous. $f$ Hered 1949;40:329-34.

21 Stein KF, MacKensen JA. Abnormal development of the thoracic skeleton in mice homozygous for the gene for loop-tail. Am $\mathcal{f}$ Anat 1957;100:205-23.

22 Morgan W. A new crooked-tail mutation involving distinct pleiotropism. F Genet 1954;52:354-73.

23 Theiler K, Stevens LC. The development of rib-fusion, a mutation in the house mouse. Am $\mathcal{F}$ Anat 1960;106:17183.

24 Johnson DR. Extra-toes: a new mutant gene causing multiple abnormalities in the mouse. $\mathcal{F}$ Embryol Exp Morphol 1967;1 7:543-81.

25 Johnson DR. Brachyphalangy, an allele of extra-toes in the mouse. Genet Res 1969;13:275-80.

26 Grüneberg $\mathrm{H}$. Genetical studies on the skeleton of the mouse. VIII. Curly-tail. $\mathcal{f}$ Genet 1954;52:52-67.

27 Seller MJ, Embury S, Polani PE, Adinolfi M. Neural tube defects in curly-tail mice. II. Effect of maternal administration of vitamin A. Proc $R$ Soc Lond [Biol] 1979;206:95-107.

28 Moase CE, Trasler DG. Delayed neural crest cell emigration from $S p$ and $S p^{d}$ mouse neural tube explants. Teratology 1990;42:171-82

$29 \mathrm{Hsu}$ CY, Van Dyke JH. An analysis of growth rates in neural epithelium of normal and spina bifidous (myeloschisis) mouse embryos. Anat Rec 1948;100:745.

Schisis) mouse embryos. Anat Rec 1948; 100:745.
30 Wilson DB. Proliferation in the neural tube of the splotch $(S p)$ mutant mouse. $\mathcal{F}$ Comp Neurol 1974;154:249-56.

31 Kapron-Brás CM, Trasler DG. Histological comparison of the effects of the splotch gene and retinoic acid on the 
closure of the mouse neural tube. Teratology 1988;37:38999.

32 O'Shea KS, Rheinheimer JST, O'Shea JM. Morphometric analysis of the forebrain anomalies in the delayed splotch mutant embryo. I Craniofac Genet Dev Biol 1987;7:35769.

33 Deol MS. Influence of the neural tube on the differentiation of the inner ear in the mammalian embryo. Nature 1966;209:219-20.

34 Morris GL, O'Shea KS. Anomalies of neuroepithelial cell associations in the splotch mutant embryo. Dev Brain Res 1983;9:408-10.

35 Dempsey EE. Neural tube defects - pathogenesis and geneteratogen interaction in the mouse. MSc thesis, McGill University, Montreal, 1981.

36 Dempsey EE, Trasler DG. Early morphological abnormalities in splotch mouse embryos and predisposition to gene- and retinoic acid-induced neural tube defects. Teratology 1983;28:461-72.

37 Kapron-Brás CM, Trasler DG. Gene-teratogen interaction and its morphological basis in retinoic acid-induced and its morphological basis in retinoic acid-in

38 Kapron-Brás CM, Trasler DG. Interaction between the splotch mutation and retinoic acid in mouse neural tube splotch mutation and retinoic acid in mouse
defects in vitro. Teratology 1988;38:165-73.

39 Copp AJ, Seller MJ, Polani PE. Neural tube development in mutant (curly-tail) and normal mouse embryos: the timing of posterior neuropore closure in vivo and in vitro. f Embryol Exp Morphol 1982;69:151-67.

40 Wilson DB, Finta LA. Gap junctional vesicles in the neura tube of the splotch $(S p)$ mutant mouse. Teratology 1979;19:337-40.

41 Wilson DB, Finta LA. Early development of the brain and spinal cord in dysraphic mice: a transmission electron microscopic study. $\mathcal{f}$ Comp Neurol 1980;190:363-71.

42 Kapron-Brás CM, Vekemans MJJ, Trasler DG. A chromosome marker for the early detection of mouse embryos carrying the neural tube defect mutation splotch. Can $\mathcal{f}$ Genet Cytol 1986;28:862-6.

43 Roderick TH. Strain distributions of genetic polymorphisms in the mouse. In: Heiniger HJ, Dorey JJ eds. Handbook on genetically standardized $\mathcal{F} A X$ mice. 3rd ed. Bar Harbor, Maine: The Jackson Laboratory, 1980: Bar Harb-2.30

44 Davisson MT, Roderick TH. Chromosomal banding patterns of two paracentric inversions in mice. Cytogenet Cel Genet 1973;12:398-403.

45 Cole WA, Trasler DG. Gene-teratogen interaction in insulin-induced mouse exencephaly. Teratology 1980;22:125 39.

46 Dagg CP. Combined action of fluorouracil and two mutan genes on limb development in the mouse. $f$ Exp Zool 1967;164:479-89.

47 Johnson DR. Trypan blue and the extra-toes locus in the mouse. Teratology 1970;3:105-10.

48 Hamburgh M, Herz R, Landa G. The effect of trypan blue on expressivity of the brachyury gene " $T$ " in mice. on expressivity of the
Teratology $1970 ; 3: 111-8$.

49 Winfield JB, Bennett D. Gene-teratogen interaction: potentiation of actinomycin $\mathrm{D}$ teratogenesis in the house mouse tiation of actinomycin $D$ teratogenesis in the house mouse
by the lethal gene brachyury. Teratology $1971 ; 4: 157-71$.

by the lethal gene brachyury. Teratology $1971 ; 4: 157-71$.
50 Trasler DG, Kemp D, Trasler TA. Increased susceptibility to 6-amino-nicotinamide-induced cleft lip of heterozygote dancer mice. Teratology 1984;29:101-4.

51 Seller MJ, Perkins KJ. Prevention of neural tube defects in curly-tail mice by maternal administration of vitamin A Prenat Diagn 1982;2:297-300.
52 Kapron-Brás CM, Trasler DG. Reduction in the frequency of neural tube defects in splotch mice by retinoic acid. Teratology 1985;32:87-92.

53 Seller MJ, Perkins KJ. Effect of mitomycin C on the neural tube defects of the curly-tail mouse. Teratology 1986;33:305-9.

54 Copp AJ, Crolla JA, Brook FA. Prevention of spinal neural tube defects in the mouse embryo by growth retardation during neurulation. Development 1988;104:297-303.

55 O'Shea KS, Liu LH. Basal lamina and extracellular matrix alterations in the caudal neural tube of the delayed splotch embryo. Dev Brain Res 1987;37:11-26.

56 McLone DG, Knepper PA. Role of complex carbohydrates and neurulation. Pediatr Neurosci 1986;12:2-9.

57 Trasler DG, Morriss-Kay GM. Immunohistochemica localization of chondroitin and heparan sulfate proteoglycans in pre-spina bifida splotch mouse embryos. Teratology 1991;44:571-9.

58 Yang XM, Trasler DG. Abnormalities of neural tube formation in pre-spina bifida splotch-delayed mouse embryos. Teratology 1991;43:643-57.

59 Moase CE, Trasler DG. N-CAM alterations in splotch neural tube defect mouse embryos. Development (in press).

60 Edelman GM, Chuong CM. Embryonic to adult conversion of neural cell adhesion molecules in normal and staggere mice. Proc Natl Acad Sci USA 1982;79:7036-40.

61 Cole GJ, Schubert D, Glaser L. Cell-substratum adhesion in chick neural retina depends upon protein-heparan sulfate interactions. $\mathcal{F}$ Cell Biol 1985;100:1192-9.

62 Hoffmann S, Edelman GM. A proteoglycan with HNK-1 antigenic determinants as a neuron-associated ligand for antigenic determinants as a neuron-associated ligand

63 Epstein DJ, Malo D, Vekemans M, Gros P. Molecular characterization of a deletion encompassing the splotch mutation on mouse chromosome 1. Genomics 1991;10:89 93.

64 Arnvig J. The syndrome of Waardenburg. Acta Genet Stat Med (Basel) 1959;9:41-6.

65 Lavergne MG. Problème d'eugénisme posé par une famille atteinte du syndrome de Waardenburg-Klein. $f$ Genet Hum 1959;10:80-5.

66 Bwibo NO, Mkono MD. Waardenburg's syndrome in an African child. Hum Hered 1970;20:19-22.

67 Roux CH, Baheux G, Gaulier M, Caldera R, Soepardan L. Une observation familiale portent sur quatre génération et vingt trois sujets. Ann Genet (Paris) 1970;13:125-8.

68 David TJ. Waardenburg's syndrome in two siblings and their parents. Hum Genet 1971;14:81-2.

69 Mehin N, Trasler DG. Possible prevention of neural tube defects in splotch-delayed $\left(\mathrm{Sp}^{\mathrm{d}} / \mathrm{Sp}^{\mathrm{d}}\right)$ by food deprivation. defects in splotch-delayed (S

70 Goulding MD, Chalepakis G, Deutsch U, Erselius JR, Gruss P. Pax-3, a novel murine DNA binding protein expressed during early neurogenesis. EMBO $\mathcal{f} 1991$ 10:1135-47.

71 Epstein DJ, Vekemans $\mathrm{M}$, Gros $\mathrm{P}$. Splotch $\left(\mathrm{Sp}^{2 \mathrm{H}}\right)$, a mutation affecting development of the mouse neural tube, shows a deletion within the paired homeo domain of Shows a deletion within the

72 Burri M, Tromvoukis Y, Bopp D, Frigerio G, Noll $M$ Conservation of the paired domain in metazoans and its structure in three isolated human genes. EMBO $\mathcal{f}$ 1989;8:1183-90. 